\title{
Statistical Atlas Based Extrapolation of CT Data
}

\author{
Gouthami Chintalapani $^{a}$, Ryan Murphy ${ }^{b}$, Robert S. Armiger $^{c}$, Jyri Lepist'o ${ }^{d}$, Yoshito Otake $^{a}$, \\ Nobuhiko Sugano $^{e}$, Russell H. Taylor ${ }^{a}$ and Mehran Armand ${ }^{c}$ \\ ${ }^{a}$ Department of Computer Science, Johns Hopkins University, Baltimore, USA; \\ ${ }^{b}$ Department of Biomedical Engineering, Johns Hopkins University, Baltimore, USA; \\ ${ }^{c}$ Johns Hopkins University Applied Physics Laboratory, Laurel, USA; \\ ${ }^{d}$ Pjhlajalinna Hospital, Tampere, Finland; \\ ${ }^{e}$ Department of Orthopedic Surgery, Osaka University Graduate School of Medicine, Japan;
}

\begin{abstract}
We present a framework to estimate the missing anatomical details from a partial CT scan with the help of statistical shape models. The motivating application is periacetabular osteotomy (PAO), a technique for treating developmental hip dysplasia, an abnormal condition of the hip socket that, if untreated, may lead to osteoarthritis. The common goals of PAO are to reduce pain, joint subluxation and improve contact pressure distribution by increasing the coverage of the femoral head by the hip socket. While current diagnosis and planning is based on radiological measurements, because of significant structural variations in dysplastic hips, a computer-assisted geometrical and biomechanical planning based on CT data is desirable to help the surgeon achieve optimal joint realignments. Most of the patients undergoing PAO are young females, hence it is usually desirable to minimize the radiation dose by scanning only the joint portion of the hip anatomy. These partial scans, however, do not provide enough information for biomechanical analysis due to missing iliac region. A statistical shape model of full pelvis anatomy is constructed from a database of CT scans. The partial volume is first aligned with the statistical atlas using an iterative affine registration, followed by a deformable registration step and the missing information is inferred from the atlas. The atlas inferences are further enhanced by the use of X-ray images of the patient, which are very common in an osteotomy procedure. The proposed method is validated with a leave-one-out analysis method. Osteotomy cuts are simulated and the effect of atlas predicted models on the actual procedure is evaluated.
\end{abstract}

Keywords: atlases, registration, hip dysplasia, CT

\section{INTRODUCTION}

Developmental hip dysplasia (DDH) or hip dysplasia is a deformation of the hip joint in which the hip socket is shallow leading to instability. Patients exhibiting DDH have an abnormal characterization of the hip joint with respect to the femoral head, which can cause considerable pain. This condition is more common in young adults and can progress to osteoarthritis if left untreated. Diagnosis for this condition relies on AP radiographs, but precise planning can be enhanced by obtaining computed tomography (CT) data around the hip joint. ${ }^{1,2}$

Periacetabular osteotomy (PAO) is one of the surgical procedures used to treat DDH. A successful PAO seeks to reduce contact pressure and stabilize the hip joint ${ }^{3}$ by realigning the hip joint and increasing coverage of the femoral head. A particular type of PAO, the Ganz (or Bernese) osteotomy, maintains the integrity of the posterior column and preserves blood flow to the acetabulum. To perform the osteotomy, four cuts are made (See Figure 1): 1) the body of the ischium is cut between the distal joint capsule and the psoas tendon; 2) the pubic ramus is separated from the acetabulum; 3 ) the acetabulum is separated along the ilium, beginning proximal to the anteroinferior iliac spine, turning toward the ischial spine on the anterior leg; and 4) the remaining osseous attachment on the ischium is cut. After making the cuts, the surgeon is free to reposition the acetabular fragment such that coverage is increased and the joint is stabilized. ${ }^{1}$

Further author information: (Send correspondence to G. Chintalapani)

G. Chintalapani: E-mail: gouthami@jhu.edu, Telephone: 14105163417

Medical Imaging 2010: Visualization, Image-Guided Procedures, and Modeling, edited by Kenneth H. Wong,

Michael I. Miga, Proc. of SPIE Vol. 7625, 762539 - @ 2010 SPIE · CCC code: 1605-7422/10/\$18 · doi: 10.1117/12.845570

Proc. of SPIE Vol. 7625 762539-1 


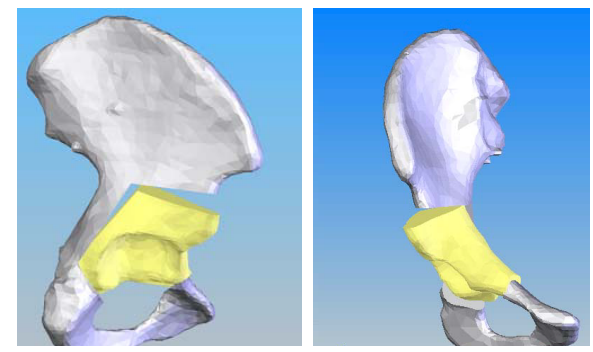

Figure 1. Example cuts in a hip osteotomy procedure

The limitations of current navigation systems that perform PAO are 1) lack of ability to track bone fragment alignment, 2) do not provide anatomical measurements, 3) do not incorporate biomechanical planning and guidance, and 4) ignore the risk of reducing joint range-of-motion. To address these limitations, some of the coauthors of this paper have developed a computer assisted biomechanical guidance system (BGS) for performing PAO ? ${ }^{?, 5}$ The BGS system performs preoperative planning and intraoperative fragment tracking to optimize the stability of the hip joint. The planning is performed on a full 3D model of the patient hip anatomy obtained through a CT scan. Ideally, a CT scan of only the hip joint is obtained, but due to the biomechanical guidance proposed in this system, the CT scan of the entire pelvis anatomy is required. The hip osteotomy patient demographic consists mainly of young (likely less than 55) females without arthritis. ${ }^{1,6}$ Excess radiation is a cause for concern among all patients, especially those in this demographic.

The BGS system requires using a full pelvis model, including osseous landmarks (i.e. the centers of the femoral heads and the L5-S1 joint), registered to the patient to perform mechanical modeling of the hip joint. Optimization based on standing loads applied through the joint yields the optimal realignment such that contact pressures are reduced. ${ }^{7,8}$ Lloads from other daily activities such as walking or sitting may also be applied in BGS, leading to a spectrum of biomechanical analyses.

In this paper, we propose a method for, and report on the validity of, using statistical atlases to help plan and perform computer assisted PAO surgeries using reduced volume preoperative CT data. Typically, a CT scan of the hip joint anatomy is obtained for preoperative planning and C-arm fluoroscopic images are used for intraoperative assessment of the bone fragment alignment. Since the PAO system requires entire pelvis anatomy for intraoperative registration of the patient to the model and L5-S1 for planning, we need to extrapolate the patient pelvis anatomical structure using prior statistical models. We propose to use a statistical atlas of full pelvis anatomy and infer the missing iliac anatomy from the prior atlas and the partial CT scan of the patient. Several statistical methods for predicting missing data have been proposed in literature. ${ }^{9-11}$ We use a linear regression imputation technique to estimate the missing data from the observed data using a prior statistical atlas.

Moreover, we use X-ray images of the patient to improve the atlas inferences by registering the inferred model to these X-ray images using a deformable $2 \mathrm{D} / 3 \mathrm{D}$ registration method. Results from leave-out validation analysis are presented. The ultimate goal of this application is to accuratly track the detached acetabular fragment during PAO. We analyze the effect of errors in acetabular fragment tracking accuracy due to registration to partial CT scans. We simulated the osteotomy cuts and measured the fragment transformations errors after for performing (simulated) PAO.

The rest of the paper is organized as follows. In Section 2, we give a brief overview of our navigation system, statistical atlas construction and its adaptation to a patient scan with multi-resolution and partial data. Experimental results to validate our proposed system are presented in Section 3, with discussion and conclusions in Section 4. 


\section{METHODS AND MATERIALS}

In this section, we outline the statistical model construction, model adaptation to a new instance, and shape inference from partial data.

\subsection{Periacetabular Osteotomy}

The BGS system can be beneficial because it tracks the acetabular fragment and performs biomechanical analysis pre- and intraoperatively. ${ }^{\text {, }}, 5$ The system requires an accurate intraoperative model-to-patient registration to accurately track the acetabular fragment through realignment. If the model-to-patient registration is inaccurate, errors in computing biomechanics and measuring the acetabular realignment occur, hampering the usefulness of BGS; thus, a full pelvic CT scan is performed preoperatively so a more accurate registration can be established and biomechanics can be computed. As shown in Figure 2, the BGS system uses an optical tracker to track the tools used during PAO and acquire points on the patient.

Initially, the surgeon digitizes a smattering of points from the ilium and the iliac crest on the operative side. These points are then registered to the model using an Unscented Kalman Filter (UKF) technique, which has been shown to be more robust than traditional Iterative Closest Point (ICP) algorithms. ${ }^{12}$ Before and after the fragment reorientation, a set of four points are digitized on the acetabular fragment defining the transformation of the fragment in the patient frame. Using the UKF registration, the fragment transformation is redefined in the model frame and the fragment reorientation has been successfully tracked. Acquiring the fragment transformation in the model frame is crucial to the BGS system so the biomechanics can be accurately computed.

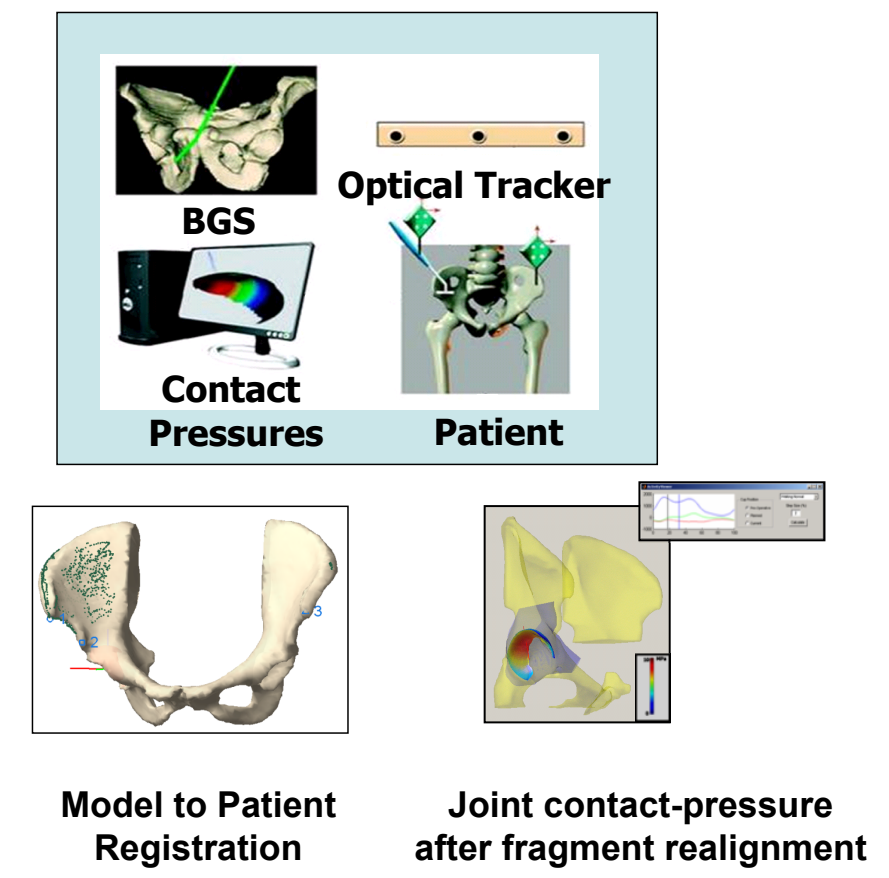

Figure 2. Various Components of Computer Assisted Periacetabular Osteotomy ${ }^{5}$

\subsection{Statistical Atlas Construction}

A statistical atlas of pelvis anatomy is constructed using the process described by Chintalapani et al. ${ }^{13}$ In general, the atlas construction process can be divided into three main steps: 1) model representation and construction, 2) model correspondences, and 3) statistical analysis. ${ }^{14}$ Our model representation consists of a tetrahedral mesh to parameterize anatomical shape and Bernstein polynomials to approximate CT intensities. ${ }^{15,16}$ Given a training data sets obtained from CT scans of patients, we select a template CT and manually segment and mesh it to 
create a template mesh. To establish point correspondences across the training data sets, we use a grayscale deformable registration method. ${ }^{17}$ All the data sets in the training sample are registered deformably to the template $\mathrm{CT}$ and the resulting deformation field is applied to the template mesh to create mesh instances for the training data. The vertices of these mesh instances define the point distribution model (PDM) for shape properties. Principal component analysis on this PDM results in a shape atlas consisting of an average shape representation and the shape variations in the form of eigenmodes. This shape model is further refined by using an iterative bootstrapping technique described in. ${ }^{13}$ Using this method, the atlas is iteratively registered to the training CT data sets and updated after each iteration resulting in stable shape variations. Although our atlas can incorporate both the shape and intensity properties, only the surface model of a patient anatomy is used for BGS procedure. The rest of the paper, therefore, uses shape statistical models only. Hence, we trim the tetrahedral meshes to extract surface mesh instances of each of the training CT data set.

A surface mesh instance is defined as a set of $3 \mathrm{D}$ points in space that define the shape of the anatomical region of interest, $s_{i}=\left[x_{1}, y_{1}, z_{1} ; x_{2}, y_{2}, z_{2} ; \ldots x_{n}, y_{n}, z_{n}\right]$. These mesh instances are aligned using procrustes alignment procedure to exclude any translation, rotation and scale from the inherent shape variations and vectorized such that $\overrightarrow{s_{i}}=\left[x_{1}, y_{1}, z_{1}, x_{2}, y_{2} \ldots . z_{n}\right]$. Given a set of such vectorized shape instances, $S=\left\{\overrightarrow{s_{1}}, \overrightarrow{s_{2}}, \ldots \overrightarrow{s_{N}}\right\}$, The average shape, $S$ can be computed as

$$
\bar{S}=\frac{1}{N} \sum_{i=1}^{N} \overrightarrow{s_{i}} ;
$$

and the covariance matrix, $C$ is

$$
C=\frac{1}{N-1}\{\Delta S\}\{\Delta S\}^{T} ; \quad \Delta S=\left\{\overrightarrow{s_{1}}-\bar{S}, \quad \overrightarrow{s_{2}}-\bar{S}, \ldots, \quad \overrightarrow{s_{N}}-\bar{S}\right\}
$$

The principal components can be obtained by taking SVD decomposition of $C$,

$$
C=U D V^{T}
$$

where $U=\left\{\vec{U}_{1}, \vec{U}_{2}, \ldots, \vec{U}_{N-1}\right\}$ are the orthogonal shape modes and $D=\operatorname{diag}\left(d_{1}, d_{2}, \ldots, d_{N-1}\right)$ are the singular values. The eigenvalues are given as $E=\left\{e_{1}=d_{1}^{2}, \quad e_{2}=d_{2}^{2}, \ldots, \quad e_{N-1}=d_{N-1}^{2}\right\}$

Given this low-dimensional parameterization of shape space, we can write any given shape instance as a linear combination of the orthogonal modes.

$$
\vec{s}=\bar{S}+\lambda * U
$$

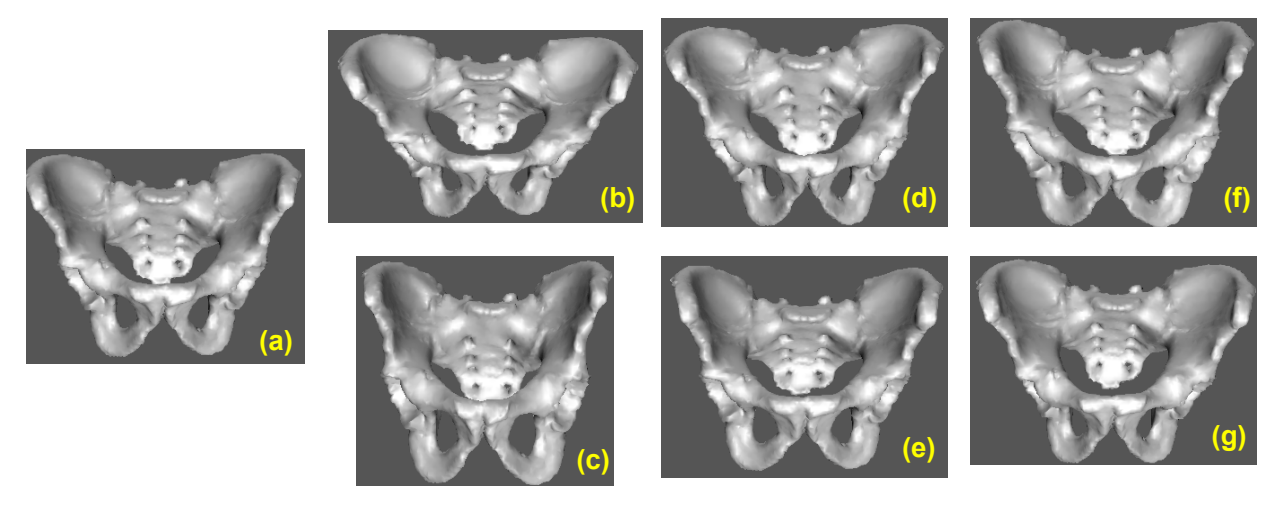

Figure 3. DRRs of mean shape and first three principal modes. (a) mean shape $(\bar{S})$; (b)-(c) Mode $1\left(\bar{S} \pm 3 e_{1} S^{1}\right)$; (d)-(e) Mode $2\left(\bar{S} \pm 3 e_{2} S^{2} ;(\mathrm{f})\right.$-(g) Mode $3\left(\bar{S} \pm 3 e_{3} S^{3}\right)$ 


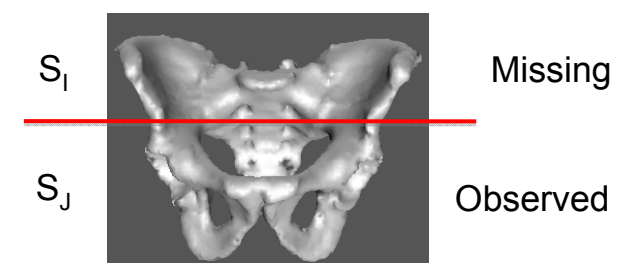

Figure 4. Figure showing the simulated observed and missing parts of pelvis anatomy for the experiments in this paper

\subsection{Atlas Adaptation}

As mentioned in Section 2.1, both biomechanical planning based on mechanical analysis and intraoperative registration require 3D pelvis models. In this section, we explore a method of obtaining an accurate patient specific preoperative model using prior information from statistical atlases.

\subsubsection{Partial CT scan}

Typically, a CT scan of the hip joint region is obtained. We then manually segment the scanned pelvis anatomy and randomly sample the segmented volume. These points are then registered to the statistical atlas to deduce the missing pelvis shape information. The registration step consists of two steps, rigid registration and mode matching. Since, the exact point correspondences between the random sample from the patient anatomy and the atlas surface mesh are not established, we use an ICP matching step to project the points on to the surface mesh of the atlas and compute the closest points on the mesh to establish valid point correspondences.

Given an atlas consisting of mean shape, $\bar{S}$, and orthogonal modes $U$, we can rearrange the matrices such that $\bar{S}=\left[\begin{array}{l}\bar{S}_{I} \\ \bar{S}_{J}\end{array}\right]$ and $U=\left[\begin{array}{l}U_{I} \\ U_{J}\end{array}\right]$, where, $\bar{S}_{J}$ is the mean of the observed data and $\bar{S}_{I}$ is the missing data. $U_{I}$ and $U_{J}$ are the corresponding mode vectors of the missing and observed data respectively (See figure 4).

From the partial CT scan of the patient data, we manually segment and extract random sample points $p^{k}, k=1,2, \ldots m$. These points are registered to the observed part of the atlas, $\overline{S_{J}}$ and $U_{J}$. The transformation between the atlas and the patient can be split into two components, rigid and deformable components which can be combined into one equation as follows:

$$
F^{t} * p^{k} \approx \bar{S}_{J}^{k}+\lambda^{t} * U_{J}^{k}, \quad k=1,2, \ldots, m
$$

There are two unknowns in the above equation: 1) $F^{t}=R^{t}, T^{t}$, the rigid transformation between the patient and the atlas; and 2) $\lambda^{t}$, the mode weight parameters that will match the atlas to the patient data. It is possible to solve for these two unknowns iteratively by fixing the transformation $F^{t}$, solving for $\lambda^{t}$, and updating the atlas mesh model and computing $F^{t+1}$. This process can be iterated until convergence. To achieve better convergence, these two steps can be linearized into one step. With the assumption that the change in the transformation $F$ is very small from iteration $t$ to $t+1$, we can write,

$$
F^{t+1}=\Delta F^{t+1} * F^{t}
$$

where

$$
\Delta F^{t+1}=\left[\Delta R\left(\vec{\alpha}^{t+1}\right), \vec{\epsilon}^{t+1}\right]=\left[I+s k\left(\vec{\alpha}^{t+1}\right), \vec{\epsilon}^{t+1}\right]
$$

Using the above equation, equation 5 can be written as

$$
\left\{F^{t} * p_{k}\right\} * \vec{\alpha}^{t+1}-\bar{\epsilon}^{t+1}+\lambda^{t+1} * U_{J}^{k} \approx F^{t} * p_{k}-\bar{S}_{J}^{k}
$$


We can form a system of linear equations for each $k$ and solve it for $\vec{\alpha}^{t+1}, \vec{\epsilon}^{t+1}, \lambda^{t+1}$. Having computed the optimal $\lambda$, we can now estimate the missing region as follows.

$$
S^{e s t}=\left[\begin{array}{c}
S_{I}^{e s t} \\
S_{J}^{e s t}
\end{array}\right]=\left[\begin{array}{c}
\bar{S}_{I} \\
\overline{S_{J}}
\end{array}\right]+\lambda\left[\begin{array}{c}
U_{I} \\
U_{J}
\end{array}\right]
$$

The extrapolated patient model $S^{\text {est }}$ consists of errors in both the observed and the missing data parts. Since we already have exact information for the observed part from the CT, we replace the predicted observed part

with the true data such that, $S^{\text {est }}=\left[\begin{array}{c}S_{I}^{\text {est }} \\ S_{J}^{\text {true }}\end{array}\right]$. We then use only the missing/predicted part of the anatomy for optimization.

\subsubsection{X-ray Guidance}

Since hip dysplasia is an abnormality of the joint region only, the rest of the pelvis anatomy may or may not be abnormal. Hence, the inferences from the statistical atlas might be error prone and some information about the missing iliac region might be helpful in predicting more accurate estimates. Fluoroscopy is very commonly used in osteotomy procedures for qualitative analysis. We propose to use intra-operative $2 \mathrm{D} \mathrm{X}$-ray images and fuse them with the 3D predicted model. We use an image based 2D/3D registration method described in. ${ }^{18}$

1. Input: X-ray images/DRRs $I_{i}$, where $i$ is the image number/view angle, camera pose parameters (intrinsic and extrinsic) $P_{i}$, mean shape $S^{0}$ and shape modes $U_{I}$, mean density $C^{0}$

2. Register the input images to the shape model, rigidly and deformably to estimate the pose and shape parameters respectively. The output consists of a pose $F(R, T, S)$ and mode weight parameters $\Phi$ such that

$$
F, \Phi=\underset{(F, \Phi)}{\operatorname{argmax}} \sum_{i} M I\left(I_{i}, \operatorname{DRR}\left(F \cdot\left(\bar{S}_{I}+\Phi U_{I}\right)\right)\right)
$$

3. Transform the shape model and create DRRs of the registered shape model with mean density and density modes.

$$
S^{\text {est }}=\left[\begin{array}{c}
S_{I}^{e s t} \\
S_{J}^{\text {true }}
\end{array}\right]=\left[\begin{array}{c}
F *\left(\bar{S}_{I}+\Phi U_{I}\right) \\
S_{J}^{\text {true }}
\end{array}\right]
$$

\section{EXPERIMENTS, RESULTS AND DISCUSSION}

From a database of 110 pelvis CT data sets, we used 104 data sets for atlas creation and six for leave-out analysis. Six data sets were selected randomly from the database and were not used in the atlas creation. Partial CT scans of these six data sets were simulated by keeping the CT slices corresponding to the hip joint region and excluding the iliac slices. These data sets were sampled randomly and the missing regions were predicted using the proposed method. The surface distance between the true iliac model and the inferred model was computed. Figure 5 shows the efficiency of atlas predictions as a function of number of principal components and the amount of scanned anatomical region. The average surface distance varied from $1.5 \mathrm{~mm}$ to $2.1 \mathrm{~mm}$ for various scan sizes.

Table 1 shows results from leave-one-out analysis for six data sets. We have simulated partial CT scans from full CT scans by masking out the slices corresponding to the iliac region (See Figure 4). For 2D/3D registration, we have used two simulated X-ray like images in orthogonal views. The extrapolated models were compared to the ground truth $\mathrm{CT}$ model. These experiments show better accuracy with X-ray images as compared to the partial CT scan only.

There are primarily two factors that affect the accuracy of our proposed method. The first factor is the atlas itself and its ability to generalize an unseen shape. This can be measured from the leave-one-out validation using all the points from the CT, which we refer to as "Full CT" in the paper. The second factor measures the ability of the proposed method to predict the missing data. The goal of these experiments is to be able to predict the missing anatomical regions such that the accuracy numbers compare to the ones obtained from full CT data. 


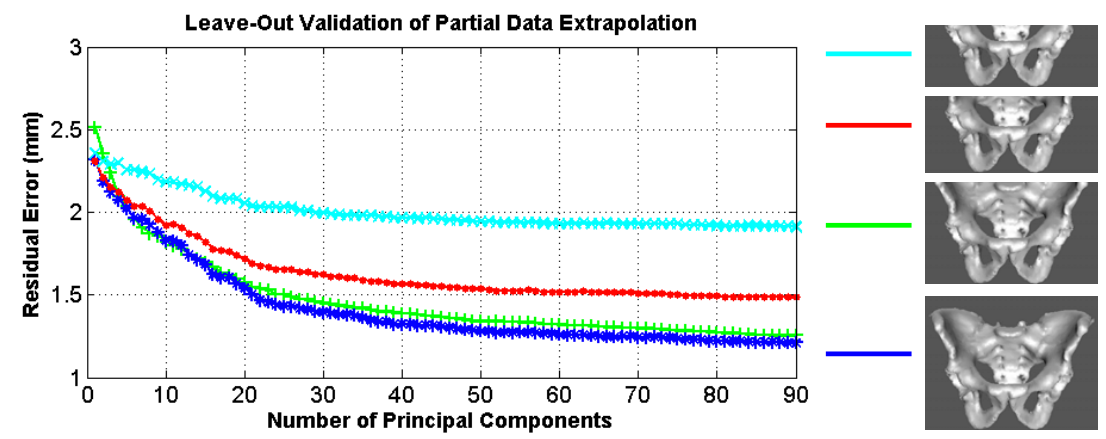

Figure 5. Leave-out analysis of proposed method for various sizes of the observed data

\begin{tabular}{|c|c|c|c|c|c|c|c|c|c|c|c|c|}
\hline \multirow{2}{*}{$\#$} & \multicolumn{4}{|c|}{ Full CT } & \multicolumn{4}{|c|}{ Partial CT } & \multicolumn{4}{|c|}{ Partial CT + X-ray } \\
\hline & mean & $\max$ & std & $95 \%$ & mean & $\max$ & std & $95 \%$ & mean & $\max$ & std & $95 \%$ \\
\hline 1 & 1.41 & 8.20 & 1.06 & 3.45 & 1.97 & 14.06 & 1.69 & 5.17 & 1.37 & 10.94 & 1.13 & 3.54 \\
\hline 2 & 1.88 & 7.25 & 1.42 & 4.71 & 2.15 & 12.25 & 1.73 & 5.28 & 1.73 & 14.78 & 1.71 & 4.51 \\
\hline 3 & 1.55 & 7.72 & 1.20 & 3.77 & 2.45 & 11.33 & 2.08 & 6.89 & 1.41 & 6.81 & 1.10 & 3.54 \\
\hline 4 & 1.32 & 5.77 & 1.01 & 3.27 & 1.69 & 9.06 & 1.43 & 4.58 & 1.21 & 6.80 & 1.03 & 3.27 \\
\hline 5 & 1.72 & 8.29 & 1.17 & 3.79 & 1.62 & 6.87 & 1.24 & 3.93 & 1.36 & 8.17 & 1.13 & 3.61 \\
\hline 6 & 1.69 & 10.58 & 1.55 & 4.78 & 2.64 & 14.87 & 2.27 & 7.18 & 1.71 & 11.33 & 1.54 & 5.06 \\
\hline avg & 1.59 & 7.96 & 1.23 & 3.96 & 2.08 & 11.40 & 1.74 & 5.50 & 1.46 & 9.80 & 1.27 & 3.92 \\
\hline
\end{tabular}

Table 1. Residual errors from leave-out-validation tests of the proposed method. "Full CT"' represents the atlas shape adaptation to the complete CT scan of the pelvis anatomy. "Partial CT"' represents the atlas shape deformed to match the partial CT scan as given in Section 2.3.1. The last four columns represent the atlas shape deformed using both the partial CT scan and X-ray guidance. For each of these scenarios, average, maximum, standard deviation and 95\% percentile error are presented. Note that the atlas extrapolations show better accuracies with both partial CT and X-ray guidance and are comparable to the approximation using full CT data

Model to patient registration: The ultimate goal is to use this atlas-based extrapolated model for registration and planning of computer assisted PAO, we simulated osteotomy cuts on both sides (left and right pelvis) for all leave-out instance. To simulate the BGS procedure, we have randomly extracted about 500 unique points from the surface of the ilium and the iliac crest from the true CT model. These points are akin to the registration points obtained using the BGS. A uniform additive Gaussian noise of up to $0.5 \mathrm{~mm}$ is added to these points to represent the sensor/tracker noise encountered in real scenarios. These points are registered rigidly to the extrapolated models using full CT, partial CT and X-ray image based extrapolated model. Assuming the true $\mathrm{CT}$ registration is the ground truth, we compute the errors between the atlas-extrapolated model registration (in the true CT frame) and the registration computed using the true CT model. Registration simulations are performed for both left and right osteotomies on the patients. The results for this analysis are presented in Table 2.

Fragment tracking: Simulating the fragment tracking runs in a similar vein to the registration simulation. Here, we pick points on a possible acetabular fragment on the operative side. These points are picked in such a way that it mimics the points chosen by the surgeon for fragment tracking. An arbitrary, but reasonable, transformation is applied to the true points to model the post-rotation points. To each set of points, tracker and modeling errors are accounted for by adding a small amount of Gaussian noise. The fragment transformation in the model frame is computed following the BGS procedure. As with the registration procedure, using the true $\mathrm{CT}$ as the ground truth, the error of the atlas-extrapolated models' ability to track the fragment is computed and presented in Table 3 .

In general, the ICP errors are greater than those exhibited in fragment tracking. This result is similar to that achieved by Armiger? during cadaver trials on the BGS system. The basis for fragment tracking occurs 


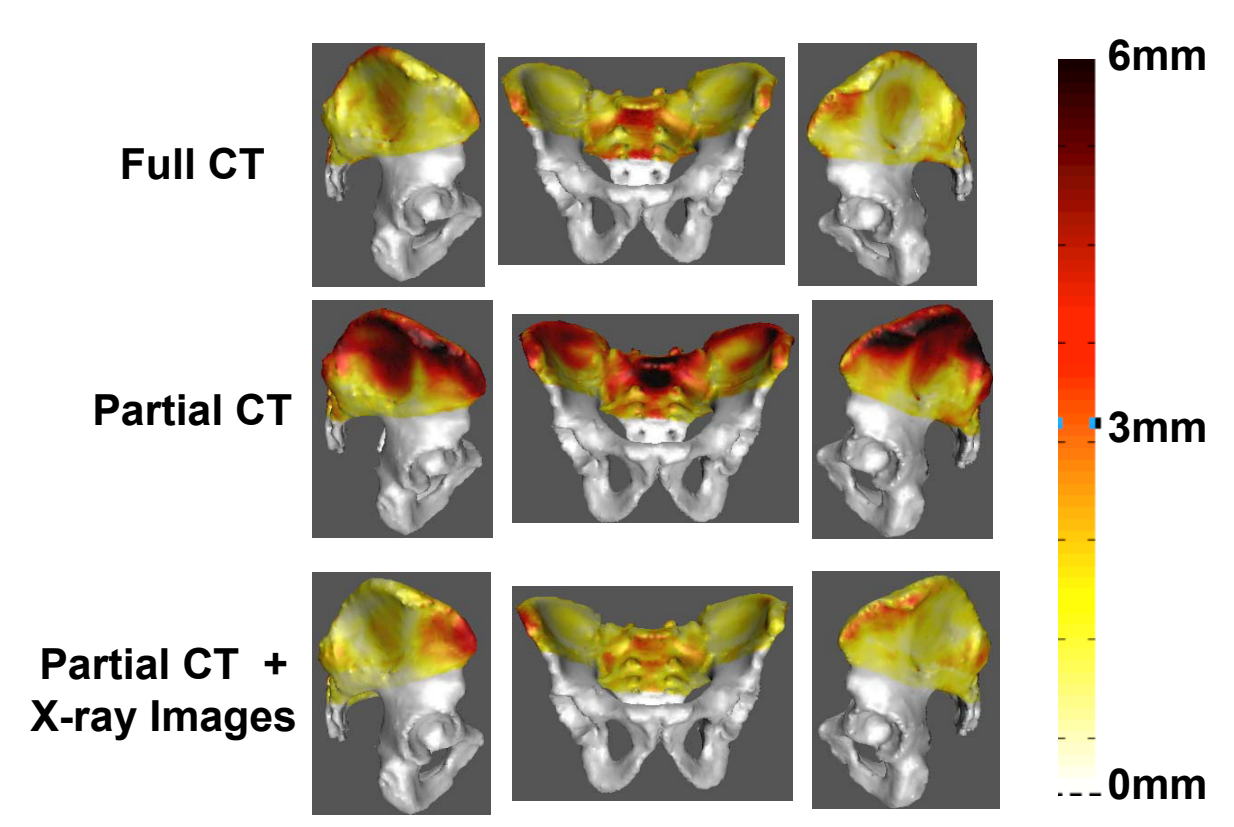

Figure 6. Distribution of surface registration errors between the atlas and full CT, partial CT and partial CT combined with X-ray images

\begin{tabular}{|c|c|c|c|c||c|c|c|c||c|c|c|c|}
\hline \multirow{2}{*}{$\#$} & \multicolumn{4}{|c||}{ Full CT } & \multicolumn{4}{c||}{ Partial CT } & \multicolumn{4}{c|}{ Partial CT + X-ray } \\
\cline { 2 - 14 } & rot & trans & mean & max & rot & trans & mean & max & rot & trans & mean & max \\
\hline & $(\circ)$ & $(\mathrm{mm})$ & $(\mathrm{mm})$ & $(\mathrm{mm})$ & $(\mathrm{\circ})$ & $(\mathrm{mm})$ & $(\mathrm{mm})$ & $(\mathrm{mm})$ & $(\mathrm{\circ})$ & $(\mathrm{mm})$ & $(\mathrm{mm})$ & $(\mathrm{mm})$ \\
\hline 1 & 2.63 & 1.03 & 1.68 & 5.86 & 4.23 & 2.17 & 2.05 & 7.55 & 2.56 & 2.73 & 1.65 & 5.86 \\
\hline 2 & 1.29 & 0.97 & 1.42 & 5.56 & 2.62 & 3.39 & 1.77 & 7.15 & 2.18 & 3.90 & 1.85 & 8.26 \\
\hline 3 & 1.66 & 3.58 & 1.46 & 5.94 & 8.37 & 6.27 & 1.87 & 6.41 & 3.06 & 3.92 & 1.50 & 5.87 \\
\hline 4 & 0.87 & 0.91 & 1.21 & 4.16 & 2.00 & 2.32 & 1.64 & 5.96 & 1.42 & 2.64 & 1.46 & 6.35 \\
\hline 5 & 1.27 & 1.09 & 0.95 & 3.68 & 4.96 & 5.87 & 1.61 & 5.47 & 2.20 & 1.87 & 1.22 & 4.53 \\
\hline 6 & 1.64 & 1.97 & 1.58 & 6.93 & 4.32 & 4.12 & 1.84 & 8.75 & 1.46 & 2.74 & 1.44 & 6.17 \\
\hline avg & 1.56 & 1.59 & 1.38 & 5.35 & 4.41 & 4.02 & 1.79 & 6.88 & 2.14 & 2.96 & 1.52 & 6.16 \\
\hline
\end{tabular}

Table 2. Model to patient ICP registration errors

in the patient (tracker) frame, which means there is no associated model error; whereas the model-to-patient registration errors include both model and tracker noise. Although the fragment transformation is subsequently transformed from patient space into model space, the errors in the model-to-patient registration will not be exacerbated.

\section{CONCLUSIONS}

In this paper, we have explored the application of statistical atlases in peri-acetabular osteotomy procedures. The prior atlas of full pelvis anatomy is fused with the preoperative CT scan of the hip joint and the intra-operative $\mathrm{X}$-ray images to predict the missing pelvis anatomy. Simulation results show that the accuracy of the atlas extrapolated model is comparable to the full CT model, and has improved when X-ray images are used. The average surface distance between the extrapolated model and the true CT is $1.46 \mathrm{~mm}$. We have also simulated osteotomy procedures on the leave-out datasets and studied the implications of the extrapolated models with respect to the patient to $\mathrm{CT}$ registration and fragment tracking. An average fragment tracking error of $0.37^{\circ}$ in rotation and $2.63 \mathrm{~mm}$ in translation is reported. 


\begin{tabular}{|c|c|c|c|c|c|c|c|c|c|c|c|c|}
\hline \multirow{3}{*}{ \# } & \multicolumn{4}{|c|}{ Full CT } & \multicolumn{4}{|c|}{ Partial CT } & \multicolumn{4}{|c|}{ Partial CT + X-ray } \\
\hline & \multicolumn{2}{|c|}{ Left } & \multicolumn{2}{|c|}{ Right } & \multicolumn{2}{|c|}{ Left } & \multicolumn{2}{|c|}{ Right } & \multicolumn{2}{|c|}{ Left } & \multicolumn{2}{|c|}{ Right } \\
\hline & $\begin{array}{l}\text { rot } \\
\text { (o) }\end{array}$ & $\begin{array}{l}\text { trans } \\
(\mathrm{mm})\end{array}$ & $\begin{array}{l}\text { rot } \\
\text { (०) }\end{array}$ & $\begin{array}{l}\text { trans } \\
(\mathrm{mm})\end{array}$ & $\begin{array}{l}\text { rot } \\
\text { (o) }\end{array}$ & $\begin{array}{l}\text { trans } \\
(\mathrm{mm})\end{array}$ & $\begin{array}{l}\text { rot } \\
(\mathrm{o})\end{array}$ & $\begin{array}{l}\text { trans } \\
(\mathrm{mm})\end{array}$ & $\begin{array}{l}\text { rot } \\
(\mathrm{o})\end{array}$ & $\begin{array}{l}\operatorname{trans} \\
\text { (mm) }\end{array}$ & $\begin{array}{l}\text { rot } \\
(\mathrm{o})\end{array}$ & $\begin{array}{l}\text { trans } \\
\text { (mm) }\end{array}$ \\
\hline 1 & 0.46 & 3.66 & 0.84 & 4.37 & 0.33 & 2.74 & 2.14 & 10.75 & 0.47 & 3.67 & 0.91 & 5.09 \\
\hline 2 & 0.38 & 2.49 & 0.32 & 1.52 & 0.52 & 3.83 & 0.48 & 2.35 & 0.20 & 1.21 & 0.24 & 0.61 \\
\hline 3 & 0.57 & 4.28 & 0.48 & 3.10 & 1.79 & 10.57 & 2.09 & 11.2 & 0.46 & 2.93 & 1.47 & 8.65 \\
\hline 4 & 0.09 & 0.58 & 0.22 & 1.55 & 0.47 & 3.41 & 0.69 & 3.69 & 0.42 & 3.53 & 0.37 & 2.09 \\
\hline 5 & 0.44 & 2.62 & 0.37 & 1.81 & 1.46 & 8.37 & 1.62 & 8.2 & 0.53 & 3.56 & 0.83 & 4.23 \\
\hline 6 & 0.36 & 1.72 & 0.47 & 2.25 & 1.52 & 11.4 & 0.71 & 4.02 & 0.16 & 0.93 & 0.25 & 0.97 \\
\hline avg & 0.38 & 2.55 & 0.45 & 2.43 & 1.01 & 6.72 & 1.28 & 6.70 & 0.37 & 2.63 & 0.67 & 3.60 \\
\hline
\end{tabular}

Table 3. Fragment registration errors

The future work will enhance the extrapolation of the missing anatomical regions, by predicting the associated variances. These variances can be incorporated into the model-to-patient registration to improve the reliability of the rigid registration in the presence of model and sensor noise. Also, it is necessary to evaluate the effect of fragment tracking errors on the mechanical analysis of the contact pressure distributions during the daily living activities. Finally, the method will be validated with cadaver experiments.

\section{ACKNOWLEDGMENTS}

This work was supported by grant R01 EB006839 awarded by NIH/NIBIB.

\section{REFERENCES}

1. R. Ganz, K. Klaue, T. S. Vinh, and J. W. Mast, "A new periacetabular osteotomy for the treatment of hip dysplasias. technique and preliminary results.," Clin Orthop Relat Res, pp. 26-36, Jul 1988.

2. K. Klaue, A. Wallin, and R. Ganz, "Ct evaluation of coverage and congruency of the hip prior to osteotomy.," Clin Orthop Relat Res, pp. 15-25, Jul 1988.

3. J. A. Hipp, N. Sugano, M. B. Millis, and S. B. Murphy, "Planning acetabular redirection osteotomies based on joint contact pressures.," Clin Orthop Relat Res , pp. 134-143, Jul 1999.

4. M. Armand, J. V. S. Lepistö, A. C. Merkle, K. Tallroth, X. Liu, R. H. Taylor, and J. Wenz, "Computeraided orthopedic surgery with near-real-time biomechanical feedback," Techincal Digest 3, Johns Hopkins Univeristy Applied Physics Laboratory, 2004.

5. J. Lepistö, M. Armand, and R. S. Armiger, "Periacetabular osteotomy in adult hip dysplasia - developing a computer aided real-time biomechanical guiding system (bgs)," Suomen Ortopedia ja Traumatologia 31, pp. 186-190, Feb 2008.

6. R. Trousdale and M. Cabanela, "Lessons learned after more than 250 periacetbaular osteotomies," Acta Orthopaedica Scandinavica 74, pp. 119-126, April 2003.

7. M. Armand, J. Lepistö, K. Tallroth, J. Elias, and E. Chao, "Outcome of periacetabular osteotomy: joint contact pressure calculation using standing ap radiographs, 12 patients followed for average 2 years.," Acta Orthop 76, pp. 303-313, Jun 2005.

8. H. Tsumura, N. Kaku, S. Ikeda, and T. Torisu, "A computer simulation of rotational acetabular osteotomy for dysplastic hip joint: does the optimal transposition of the acetabular fragment exist?," $J$ Orthop Sci 10(2), pp. 145-151, 2005.

9. R. Little and D. Rubin, "'statistical analysis with missing data"," 1987.

10. B. Grung and R. Manne, "" missing values in principal component analysis"," Chemometrics and Intelligent Laboratory Systems 42, pp. 125-139, 1998.

11. M. Tipping and C. Bishop, ""probabilistic principal component analysis"," 1999.

12. M. H. Moghari and P. Abolmaesumi, "A novel incremental technique for ultrasound to ct bone surface registration using unscented kalman filtering.," Med Image Comput Comput Assist Interv 8(Pt 2), pp. 197$204,2005$. 
13. G. Chintalapani, L. Ellingsen, O. Sadowsky, J. Prince, and R. Taylor, "Statistical atlases of bone anatomy: Construction, iterative improvement and validation," in MICCAI, 4791/2007, pp. 499-506, 2007.

14. T. Cootes, C. Taylor, D. Cooper, and J. Graham, "" active shape models - their training and application"," Computer Vision Image Understanding 61(1), pp. 38-59, 1995.

15. J. Yao, A Statistical Bone Density Atlas and Deformable Medical Image Registration. PhD thesis, The Johns Hopkins University, 2002.

16. O. Sadowsky, Image Registration and Hybrid Volume Reconstruction of Bone Anatomy Using a Statistical Shape Atlas. PhD thesis, The Johns Hopkins University, 2008.

17. L. Ellingsen and J. Prince, "Deformable registration of ct pelvis images using mjolnir," in IEEE 7th Nordic Signal Processing Symposium (NORSIG), 2006.

18. O. Sadowsky, G. Chintalapani, and R. Taylor, "Deformable 2d-3d registration of the pelvis with a limited field of view, using shape statistics," in MICCAI, 4791/2007, pp. 519-526, 2007. 